
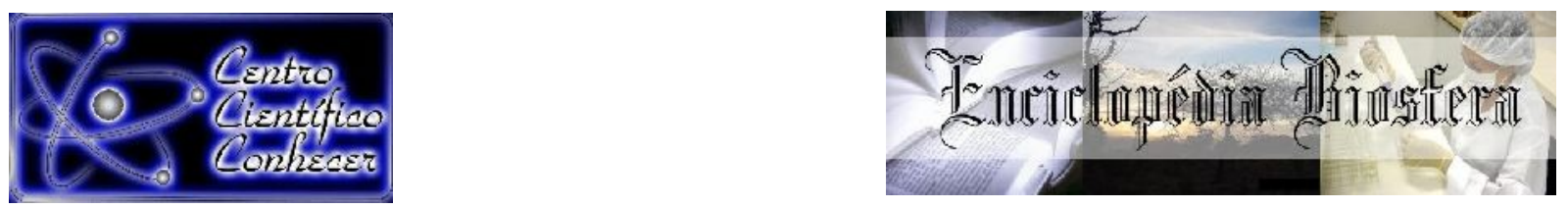

\title{
ECOLOGIA DE MICROFUNGOS AMBIENTAIS ENCONTRADOS EM SUBSTRATOS DE MANGUEZAL
}

\author{
Érica dos Anjos Almeida ${ }^{1}$, Jorge Luiz Fortuna ${ }^{2}$ \\ ${ }^{1}$ Especialista em Biociências e Biodiversidade: Ecologia e Conservação Ambiental \\ (BIOECOA) pela Universidade do Estado da Bahia (UNEB), Campus X, Teixeira de \\ Freitas-BA. Licenciada em Ciências Biológicas pela UNEB. \\ E-mail: almeidaerica5@gmail.com \\ 2 Professor Adjunto da área de Microbiologia do curso de Ciências Biológicas da \\ Universidade do Estado da Bahia (UNEB), Campus X, Teixeira de Freitas-BA. \\ E-mail: magoofortuna@gmail.com
}
Recebido em: 15/02/2021 - Aprovado em: 15/03/2021 - Publicado em: 30/03/2021
DOI: 10.18677/EnciBio_2021A8

\begin{abstract}
RESUMO
Os fungos são encontrados em toda parte, inclusive em lugares inóspitos. Os estudos desses seres são recentes na região do Extremo Sul da Bahia, portanto, há muito que ser investigado sobre estes. O manguezal é um ecossistema rico que apesar de estar sendo devastado por ações antrópicas ainda possui uma biodiversidade de hifomicetos, que podem ser desconhecidos na região. Portanto, este trabalho teve como objetivo identificar espécies de microfungos encontrados em substratos de ecossistema de Manguezal no Extremo Sul da Bahia. As coletas foram realizadas de janeiro de 2019 a janeiro de 2020 totalizando três coletas. Os substratos foram coletados em três áreas diferentes (preservada; estressada e antropizada). As análises foram realizadas no Laboratório de Biologia dos Fungos do Campus X da Universidade do Estado da Bahia (UNEB). Foram analisados 19 espécimes no qual foram identificados dez em nível de gênero, três em nível de espécies e seis não foram identificados. Os cálculos de constância identificaram que a maioria das espécies se classifica como acessória e Bipolaris sp. 1 é uma espécie constante. Verificou-se pouca similaridade entre as áreas estudadas e uma diversidade total de 2,7.
\end{abstract}

PALAVRAS-CHAVE: Hifomiceto; Restinga; Mata Atlântica.

\section{ENVIRONMENTAL MICROFUNGI FOUND IN MANGROVE SUBSTRATES}

\begin{abstract}
Fungi are found everywhere, even in inhospitable places. The studies of these beings are recent in the Southern Bahia, therefore, there is much to be investigated about them. The mangrove is a rich ecosystem that despite being devastated by human activities also has a hyphomycetes of biodiversity, which may be unknown in the region. Therefore, this work aimed to identify species of microfungi found in substrates of the mangrove ecosystem in the Southern Bahia. Samples were collected from January 2019
\end{abstract}


to January 2020 a total of three collections. The substrates were collected in three different areas (preserved, stressed and anthropized). The analyzes were carried out at the Fungi Biology Laboratory of Campus X of the State University of Bahia (UNEB). Nineteen specimens were analyzed in which ten were identified at the genus level, three at the species level and six were not identified. Constancy calculations identified that most species are classified as accessory and Bipolaris sp. 1 is a constant species. There was little similarity between the areas studied and a total diversity of 2.7.

KEYWORDS: Hyphomycetes; Sandbank; Atlantic Forest.

\section{INTRODUÇÃO}

O manguezal é um ecossistema costeiro de transição entre os ambientes terrestre e marinho, característico de regiões tropicais e subtropicais, sujeito ao regime das marés. Possui espécies vegetais bastante características, adaptadas à flutuação de salinidade e caracterizadas por colonizarem sedimentos predominantemente lodosos, com baixos teores de oxigênio (SCHAEFFER-NOVELLI, 1995).

No ano de 2000 havia $83.495 \mathrm{~km}^{2}$ de manguezal no planeta, isto é, apenas $0,2 \%$ de florestas na Terra. Este bioma é encontrado em 105 países, sendo Indonésia, Brasil, Malásia e Papua Nova Guiné concentram 50\% da área total de manguezal no mundo. Porém, o Instituto Internacional para Sustentabilidade mostrou que a área total de manguezais no mundo diminui para um total de $81.800 \mathrm{~km}^{2}$, em 2017 (LACERDA; JAKOVAC, 2020).

Ecossistema manguezal é uma parte importante para a região costeira, pois neste local ocorrem trocas de nutrientes e detritos em geomorfologia e hidrodinâmica, fornecendo biótipos de viveiro para aves marinhas, peixes, abrigando alta diversidade de microrganismos e também oferecendo diversos serviços ecológicos e bens econômicos, como madeira, peixes e mariscos (EWEL et al., 1998; KATHIRESAN, 2000; NAGELKERKEN et al., 2000; ALONGI, 2002; SHEARER et al., 2007; CHENG et al., 2009; DEBBAB et al., 2013). Também protege o litoral contra eventos de chuva intensa; reduz processos erosivos; mantém a qualidade microbiológica e físico-química da água; regula o clima local e mantém o estoque de espécies importantes para a comunidade pesqueira, gerando renda direta e indiretamente à comunidade local, além de manter atividades de turismo e manejo pesqueiro sustentável (LACERDA; JAKOVAC, 2020).

Os manguezais são um dos ecossistemas mais produtivos do planeta, e sua importância para a manutenção de bens e serviços é enorme. Os manguezais são importantes sequestradores e estocadores de carbono na biomassa e no solo. $O$ processo de sequestro de carbono por área de florestas de mangue é da mesma ordem de grandeza do observado em outras florestas tropicais úmidas. Os manguezais ainda contribuem com a redução da vulnerabilidade da zona costeira às mudanças climáticas (BRASIL, 2018).

De acordo com Lacerda e Jakovac (2020), nas florestas terrestres, o carbono está armazenado principalmente nas madeiras, porém, no manguezal o carbono se acumula, quase que totalmente no solo (50\% a $90 \%)$, devido a baixa taxa de decomposição da matéria orgânica e os períodos de inundação, aprisionando assim o carbono no solo. 
No meio ambiente os fungos desempenham papel importante na cadeia alimentar, por decomporem matéria orgânica morta, reciclando elementos vitais. A maioria das plantas depende de simbioses com fungos, conhecidas como micorrizas, para auxiliar suas raízes a absorverem minerais e água do solo. Outra importância dos fungos, para o meio ambiente, é a ciclagem de nutrientes, entre outras infinitas aplicações em prol da humanidade (GUSMÃO, 2011).

O estudo sobre fungos vem crescendo no Brasil e identificá-los permite conhecer a variedade de espécies, sua importância econômica, patológica e ambiental, principalmente dos conhecidos como fungos anamórficos ou microfungos. Além disso, Campos et al. (2005) descrevem que o conhecimento desta diversidade fúngica e funcional do manguezal é de grande importância para conhecer e entender a dinâmica de cadeias e teias envolvidas neste sistema.

Os fungos são cosmopolitas e geralmente não apresentam especificidade por hospedeiro. Sabe-se que diferentes tipos estão associados a esse ecossistema, como epífitos, sapróbios e patógenos, em diferentes substratos, tais como folhas, caules, frutos e solo. A maioria dos fungos isolados em manguezais tropicais e subtropicais corresponde aos Basidiomycota, Ascomycota e fungos anamórficos. Em comparação com os dados de produtividade primária, o conhecimento sobre os fungos de manguezais está apenas no início, podendo ser encontradas várias espécies de fungos (JONES; ALIAS, 1997).

Os estudos sobre microfungos são recentes e ainda são poucos na região do Extremo Sul da Bahia, sendo estes os mais recentes: microfungos em fragmento de Mata Atlântica no Extremo Sul da Bahia (ALMEIDA et al., 2020); identificação de microfungos em fitotelmata de bromélias no fragmento de Mata Atlântica no Extremo Sul da Bahia (OLIVEIRA; FORTUNA, 2020); microfungos associados a substratos em fitotelmata de bromélias de um fragmento de Mata Atlântica no Extremo Sul da Bahia (SILVA; FORTUNA, 2019); e comunidade fúngica endofítica e epifítica de bromélias em um fragmento de Mata Atlântica no Extremo Sul da Bahia (VIANA; FORTUNA, 2020).

Portanto, há muito que ser investigado sobre os microfungos na região do Extremo Sul da Bahia. Apesar de estar sendo devastado por ações antrópicas, como afirma Alongi (2002), que as principais razões para a destruição do manguezal são o desenvolvimento urbano, aquicultura, mineração e exploração excessiva de madeira, peixes e crustáceos, este mesmo autor ainda afirma que o manguezal possui características que, no total, são estrutural e funcionalmente únicos.

A identificação de microfungos em manguezal possibilita maiores conhecimentos sobre a biodiversidade desse ecossistema, como também serve como bioindicador para investigações futuras, tanto para a área de conservação e ecologia como para a área de evolução e genética, esta por sua vez podendo averiguar o processo de adaptação dos microrganismos neste Bioma que é conhecido como um ambiente de transição.

Sendo assim, de acordo com o descrito anteriormente, esta pesquisa teve como objetivo isolar e identificar microfungos de substratos encontrados no solo de manguezal localizado no município de Alcobaça-BA, Extremo Sul da Bahia, além de realizar análises ecológicas em relação as espécies encontradas e as respectivas áreas de coleta. 


\section{MATERIAL E METODOS}

No período de janeiro de 2019 a janeiro de 2020 foram realizadas três coletas de diferentes substratos (galhos; gravetos; folhas; cascas; ecdises, etc.), na área do ecossistema Manguezal que pertence ao domínio ecológico da Mata Atlântica, no município de Alcobaça, Extremo Sul da Bahia. A cidade possui uma extensa área de Manguezal e localiza-se entre a latitude $17^{\circ} 32^{\prime} 51,0^{\prime \prime S}$ e longitude $39^{\circ} 11^{\prime} 34,5^{\prime \prime O}$ à margem esquerda do rio Alcobaça (SILVA, 2018).

As áreas onde foram feitas as coletas foram identificadas como área $\mathbf{A}$; área $\mathbf{B}$ (margem direita do rio Alcobaça) e área $\mathbf{C}$ (margem esquerda do rio Alcobaça), localizadas na foz do rio Alcobaça (Figura 1). A classificação das áreas foi realizada de acordo com o nível de preservação, além da visualização das principais características de manguezal encontradas nestas respectivas áreas. A área preservada apresentava todas as principais características de manguezal, tais como solo enlameado devido as marés; presença de fauna típica (aves e crustáceos) e presença de vegetação típica (mangue-vermelho; mangue-branco). De acordo com diminuição e/ou ausência de uma ou mais características foi feita a classificação das outras áreas (estressada e antropizada), respectivamente.

FIGURA 1. Localização do município de Alcobaça-BA e das áreas de coletas

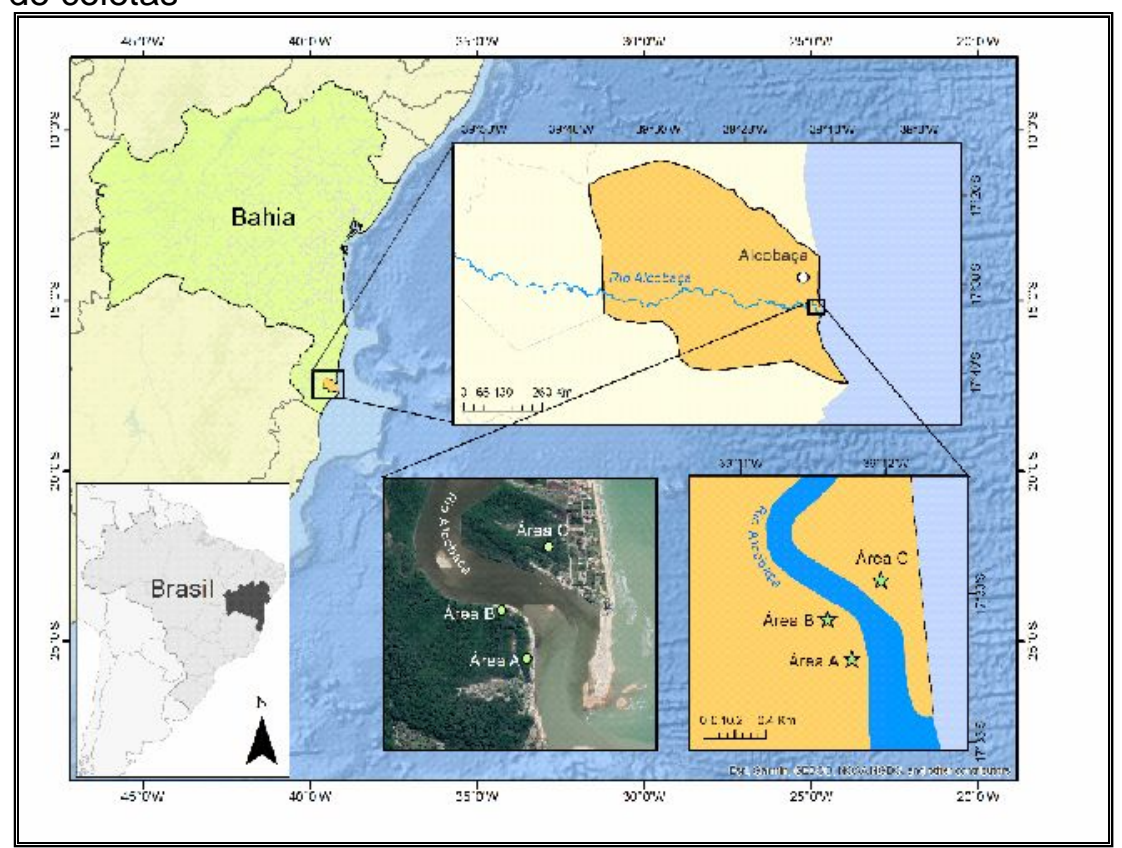

Fonte: Autores.

A área A (área preservada), encontra-se na latitude $17^{\circ} 33^{\prime} 13^{\prime \prime} S$ e longitude $39^{\circ} 11^{\prime} 37^{\prime \prime} \mathrm{O}$, com temperatura que variou entre $30^{\circ} \mathrm{C}$ a $31,8^{\circ} \mathrm{C}$ e umidade entre $58 \%$ a $68 \%$. Essa área não mostra muito dano ambiental, solo úmido sem mau cheiro, árvores típicas de manguezal e com muitos indivíduos da fauna local.

A área B (área estressada), encontra-se na latitude 17³3'05”S e longitude 
$39^{\circ} 11^{\prime} 42^{\prime \prime} \mathrm{O}$ a temperatura variou entre $29,6^{\circ} \mathrm{C}$ a $31,6^{\circ} \mathrm{C}$ e umidade entre $59 \%$ a $68 \%$. A área apresentava espécies de flora maiores (árvores) e não típicas de manguezal que as áreas $\mathbf{A}$ e $\mathbf{C}$, havia mau cheiro, sendo encontrado muito lixo sólido (peças de roupas, papeis, plásticos, vidro, alumínio, entre outros), solo mais encharcado que a área $\mathbf{A}$ e a área $\mathbf{C}$ e menos espécies de fauna.

A área C (área antropizada), encontra-se na latitude $17^{\circ} 32^{\prime} 57^{\prime \prime S}$ e longitude $39^{\circ} 11^{\prime} 31^{\prime \prime} \mathrm{O}$ a temperatura variou entre $35,1^{\circ} \mathrm{C}$ a $35,9^{\circ} \mathrm{C}$, umidade entre $49 \%$ e $50 \%$. Essa área é mais próxima da comunidade, sendo bastante antropizada, com solo menos encharcado, quase seco, árvores características de manguezal, mas já com espécies de árvores invasoras (castanheiras) e poucas espécies de fauna.

Todo o material coletado foi acondicionado individualmente em sacos plásticos identificados com letras da área e transportados imediatamente para o Laboratório de Biologia dos Fungos do Campus X da Universidade do Estado da Bahia (UNEB).

As partículas e sedimentos indesejáveis são eliminados com água da torneira. É importante que o jato de água da torneira não atinja diretamente os materiais vegetais porque, em alguns casos, o material é fracamente colonizado pelas hifas dos microfungos. Deve ser suficiente que um fluxo de água passe por toda a amostra por 20-45 minutos para lavar as partículas superficiais do solo, protozoários, nematoides, insetos e outros organismos nas amostras (CASTAÑEDA et al., 2016).

Seguindo o protocolo descrito por Gusmão e Grandi (1997), após a lavagem, os substratos passaram por um período de secagem em temperatura ambiente e depois distribuídos em placas de Petri contendo papel toalha úmido, previamente esterilizadas. As placas foram incubadas em caixa isotérmica úmida em temperatura ambiente por, no mínimo, 72 horas. Após este tempo deu-se início a observação do material utilizando-se estereomicroscópio (lupa) para localizar o crescimento de microfungos no substrato e iniciar a identificação das espécies. Esse material foi armazenado por até 40 dias e durante esse tempo ocorreu à observação do crescimento dos microfungos.

Os microfungos encontrados foram fotografados diretamente no substrato, com a visualização em estereomicroscópio, de seu ambiente natural (cascas de árvore, gravetos, ecdise, folhas, etc.). Após o registro fotográfico do microfungo, no substrato, este é retirado com o auxílio de uma agulha de insulina e colocado em lâmina de vidro com resina PVL (15 g álcool polivinílico $+22 \mathrm{~mL}$ ácido lático $+22 \mathrm{~mL}$ fenol $+100 \mathrm{~mL}$ água deionizada) no qual o fungo é fixado em lâmina com uma lamínula, a utilização da resina é para que mantenha a durabilidade das estruturas do microfungo na lâmina.

Foi realizado o registro fotográfico do fungo na lâmina com a visualização em microscópico de suas estruturas, com medição micrométrica escalar. Também foram realizados microcultivos dos microfungos para a confecção de lâminas de microscopia a fim de caracterizar as estruturas microscópicas dos fungos, tais como conídios, conidióforos, células conidiogênicas e hifas.

O cultivo dos microfungos ocorreu em placas com Ágar Cenoura Milho (CMA), segundo Almeida et al. (2020). As placas de Petri contendo CMA cultivadas com os microfungos ficaram em temperatura ambiente, em processo de esporulação durante 72 h. Depois os microfungos foram transferidos para tubos de ensaio contendo CMA, mantendo-os sob a mesma temperatura e tempo. Após a esporulação foi adicionado aos tubos óleo mineral esterilizado, o que possibilita a conservação e inibição do 
crescimento das estruturas anatômicas dos espécimes a serem analisados. Outro método também utilizado para conservação foi o método de Castellani, no qual os microfungos foram conservados em água destilada (CASTELLANI, 1939).

Os microfungos foram identificados utilizando comparação morfológica de estruturas de importância taxonômica, tais como: conídios, células conidiogênicas, conidióforos, entre outros. A identificação microscópica é realizada de acordo a taxonomia dos grupos por meio de registros bibliográficos (GUSMÃO; GRANDI, 1997; CAl et al., 2006; DUAN et al., 2007; SEIFERT et al., 2011; WIJAYAWARDENE et al., 2016). As espécies que não foram identificadas continuam na coleção para futura identificação.

As lâminas confeccionadas e identificadas, assim como os tubos de ensaio inoculados pelos microfungos, foram preservados, registrados e depositados na Micoteca do Laboratório de Biologia dos Fungos do Campus $X$ da UNEB. Foram realizadas as seguintes análises ecológicas sem réplica: riqueza de espécie $(R)$; constância (C\%); índice de similaridade de Sorensen (IS); medida de diversidade $\beta$ de Whittakker $\left(\beta_{\mathrm{w}}\right)$ e diversidade total $\left(\beta_{\mathrm{Wt}}\right)$. A riqueza de espécie foi determinada pelo número total de espécies de microfungos encontradas nas áreas estudadas (BEGON et al., 2007; BROWER et al., 1998; RICKLEFS, 2003). A constância foi calculada relacionando o número de coletas em que a espécie do microfungo foi registrada, seguindo a seguinte fórmula: $\mathrm{C} \%=\mathrm{p} / \mathrm{P} \times 100$, onde $\mathrm{C} \%=$ análise de constância $(\%) ; \mathrm{p}$ $=$ número de vezes (coletas) que a espécie foi encontrada; e $\mathrm{P}=$ número total de coletas realizadas (DAJOZ, 1978; ODUM, 1988; 2001; PINTO-COELHO, 2000).

O índice de similaridade utilizado foi o coeficiente de Sorensen (SS), cuja fórmula segue descrita: $S S=2 c /(A+B) \times 100$, onde $S S=$ índice de similaridade de Sorensen; $\mathrm{C}=$ número de espécies em comum nas duas áreas; $\mathrm{A}=$ número total de espécies de microfungos em local $A$; e $B=$ número total de espécies de microfungos em local $B$ (DAJOZ, 1978; ODUM, 1988; 2001; MULLER-DOMBOIS, 1981). Para a medida de diversidade de Whittakker utilizou-se a seguinte fórmula: $\beta_{W}=(S / \alpha)-1$, onde $\beta_{W}=$ diversidade $\beta$ de Whittakker; $S=$ número total de espécies de duas áreas; e $\alpha=$ riqueza de espécies médias das duas áreas. Já para a medida de diversidade total $\left(\beta_{W_{t}}\right)$ foi usado a seguinte fórmula: $\beta_{\mathrm{Wt}}=S / \alpha$, onde $\beta_{\mathrm{Wt}}=$ medida de diversidade total; $S=$ riqueza total de espécies de todas as áreas; e $\alpha=$ média de espécies de todas as áreas (MAGURRAN, 2013).

\section{RESULTADOS E DISCUSSÃO}

Foram analisados e processados 19 espécimes de microfungos, sendo que dez foram identificados em nível de gênero, três em nível de espécie e seis não foram identificados. Os microfungos identificados foram encontrados associados a substratos dispersos no solo do manguezal como folhas, flores, frutos, gravetos e cascas/fustes (Quadro 1). 
QUADRO 1. Lista de microfungos identificados nos quatro diferentes tipos de substratos analisados.

\begin{tabular}{|c|c|}
\hline SUBSTRATOS & MICROFUNGOS \\
\hline Folha & Monodictys sp.; Zygosporium mycophilum \\
\hline Flor & Monodictys sp. \\
\hline Graveto & Bipolaris sp. 1; Bipolaris croanotis; Nectria sp.; Bactrodesmium sp.; Não \\
& Identificado 3; Não Identificado 4; Não Identificado 5 \\
\hline Casca/Fuste & Helicoma sp.; Bipolaris sp. 1; Bipolaris sp. 2; Não Identificado 1 \\
\hline Frutos & Aspergillus niger; Aspergillus sp.; Pestalotia sp.; Não Identificado 2, \\
\hline
\end{tabular}

Dos 13 espécimes analisados foram identificados os seguintes microfungos: Aspergillus niger, Aspergillus sp.; Bipolaris crotonis; Bipolaris sp. 1; Bipolaris sp. 2; Pestalotia sp.; Nectria sp.; Monodictys sp.; Helicosporium sp.; Zygosporium mycophilum; Bactrodesmium sp. (Tabela 1).

TABELA 1. Microfungos identificados nas áreas de estudo do manguezal de Alcobaça-BA.

\begin{tabular}{cc}
\hline MICROFUNGOS & NÚMERO DE ESPÉCIMES \\
\hline Aspergillus niger & 1 \\
Aspergillus sp. & 1 \\
Bipolaris crotonis & 1 \\
Bipolaris sp. 1 & 2 \\
Bipolaris sp. 2 & 1 \\
Bactrodesmium sp. & 1 \\
Helicosporium sp. & 1 \\
Monodictys sp. & 2 \\
Nectria sp. & 1 \\
Pestalotia sp. & 1 \\
Zygosporium & 1 \\
mycophilum. & 2 \\
Não Identificado 1 (NI 1) & 1 \\
Não Identificado 2 (NI 2) & 1 \\
Não Identificado 3 (NI 3) & 1 \\
Não Identificado 4 (NI 4) & 1 \\
Não Identificado 5 (NI 5) & $\mathbf{1 9}$ \\
\hline TOTAL &
\end{tabular}

\section{MICROFUNGOS IDENTIFICADOS Aspergillus}

Os fungos do gênero Aspergillus apresentam um talo filamentoso constituído por hifas septadas, com ramificações dicotômicas fazendo ângulos de $45^{\circ}$, mostrando estruturas de reprodução assexuada situadas no cume de uma vesícula terminal com forma variável, que é o prolongamento do conidióforo. Colônias efundidas, de várias cores, frequentemente verdes ou amareladas, às vezes marrons ou pretas (CARVALHO, 2013). Micélio parcialmente imerso, parcialmente superficial, cerdas e hifópodes ausentes (ELLIS, 1971).

Aspergillus niger carateriza-se por desenvolver colônias em menos de sete dias, apresentando no início aspecto de camurça branca ou amarelada, mas que rapidamente fica coberta por uma densa camada de cabeças conidiais negras 
(MARTINS et al, 2005). Tanto Aspergillus sp. quanto Aspergillus niger encontrados na coleta apresentam conídios de 2,5 $\mathrm{m}$ com hifas cenocíticas, o primeiro é hialino e ambos foram encontrados em fruto (Figura 2).

\section{Bipolaris}

O gênero Bipolaris inclui importantes patógenos de plantas com distribuição mundial. O reconhecimento de espécies no gênero tem sido incerto devido à falta de dados moleculares de culturas de ex-tipo, bem como características morfológicas sobrepostas (MANAMGODA, 2014).

Apresentam conidióforos marrons, em sua maioria simples, produzindo conídios pelo poro apical, retomando o crescimento sipodialmente e formando conídios em novas pontas sucessivas, conídios marrons, multicelulares, elípticos, retos ou curvos, germinando por um tubo germinativo em cada extremidade, parasita, principalmente em gramíneas (BARNETT; HUNTER, 1998).

Em ambos os fungos encontrados apresentavam conídios de aproximadamente de 12 a 17,5 $\mu \mathrm{m}$ de largura e 25 a $37,5 \mu \mathrm{m}$ de comprimento. O que diferenciara entre esses espécimes foi o formato dos septos e cores. Bipolaris crotonis é hialino com septos desregulares encontrado em um picnídio. Enquanto que Bipolaris sp. 1 e Bipolaris sp. 2 são da cor marrom com septos, porém os septos entre estes são diferenciados, ambos imersos em himênio (Figura 2).

\section{Bactrodesmium}

Apresentam colônias esporodoquiais, escalonadas, puntiformes, marrons ou pretas. Micélio principalmente imerso, consistindo em hifas ramificadas, septadas, lisas, de paredes finas, de marrom claro a marrom escuro (CAl et al., 2006). O gênero Bactrodesmium é um fungo sapróbio, encontrado em água, folhedo, tronco em decomposição, naturalizado, recorrente no Nordeste do País, com registros na Bahia, Ceará e Paraíba, com domínio na Caatinga (GUSMÃO; PEFNNING, 2015). O espécime encontrado apresentava conídio de forma oval, com três septos, no qual a base é marrom e depois escurece ficando negro, hifas cenocíticas e negras (Figura 2).

\section{Monodictys}

Colônias com efusão, verde, azul esverdeado, lavanda, marrom-escuro ou preto. Micélio principalmente superficial, ou parcialmente imerso, consistindo de hifas ramificadas, septadas, lisas ou verrucosas, de paredes finas, de cor clara a marrom média (CAl et al., 2006).Os espécimes encontrados têm o conídio de cor negra com conídios de aproximadamente 25-37,5 $\mu \mathrm{m}$ de largura e comprimento (Figura 3).

\section{Helicoma}

Colônias efundidas, peludas ou aveludadas, amareladas, marrom-acinzentadas a marrom ou oliváceas. Micélio parcialmente imerso, parcialmente superficial, consistindo de hifas ramificadas, septadas, lisas, de paredes finas, sub-hialinas a marrom claro (CAI et al.,2006). O espécime encontrado tem conídios de cor amarela, com aproximadamente sete septos, hifas cenocíticas e de cor amarronzada (Figura 3). 


\section{Nectria}

Ascomata peritecióide, superficial ou parcialmente imerso em um estroma, glose, subglobose ou obpiriforme, coriáceo, amarelo, vermelho a vermelho escuro, papilado, ostiolado, solitário a gregário liso, ocasionalmente com verrugas. Ostíolo principalmente central, curto, de cores vivas, perifisado (CAl et al., 2006). O espécime encontrado tem um conídio de comprimento de $65 \mu \mathrm{m}$ acinzentado com um septo e com peritécio escuro (Figura 3).

FIGURA 2. Aspergillus niger. (A) conidioma (cm); hifa (h); (B) conídios (c). (C) A. niger no substrato. Aspergillus sp.: (D-F) conidioma (cm); hifa (h); (E) Aspergillus sp. no substrato. Bipolaris croanotis: (G-H) conídios (c); (I) B. croanotis no substrato; picnídio (p). Bipolaris sp. 1: (J) himênio $(\mathrm{hm})$; (K) conídio (c); (L) picnídio (p). Bipolaris sp. 2: (M) picnídio (p); (N) conídio (c).

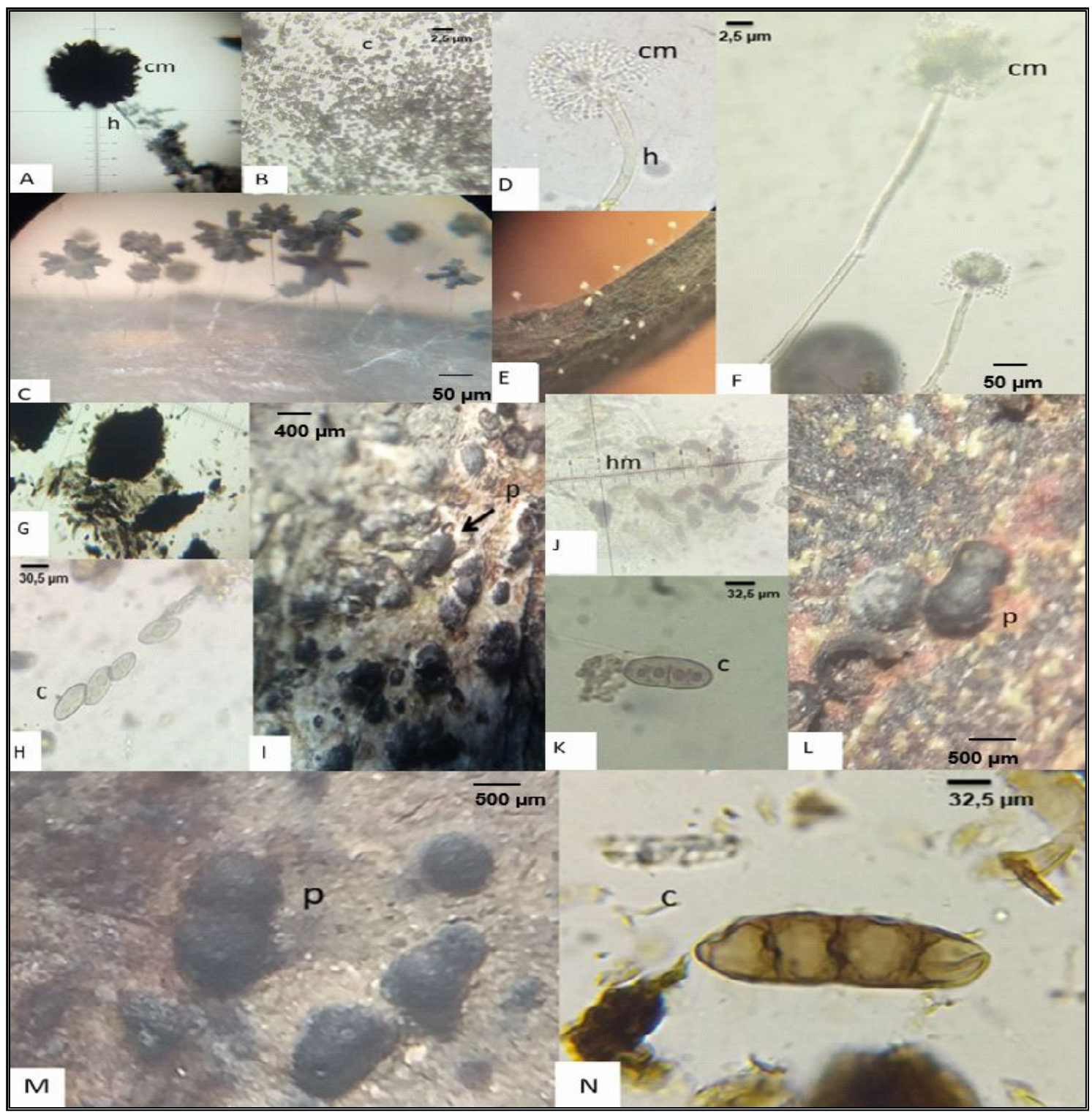

ENCICLOPÉDIA BIOSFERA, Centro Científico Conhecer - Jandaia-GO, v.18 n.35; p. 104 
FIGURA 3. Bactrodesmium sp.: (A) no substrato; (B-C) conídios (c); hifa (h). Helicoma sp.: (D) no substrato; (E-F) conídios (c); hifa (h). Pestalotia sp.: (G) conídios (c); (H) picnídio (p). Nectria sp.: (I) himênio (hm); conídios (c); (J) picnídio (p). Zygosporium mycophilium: (K) no substrato; (L) hifas; (M) conidióforo vesicular (cv); conídio (c). Monodictys sp.: (N) no substrato; (O) conídios (C).

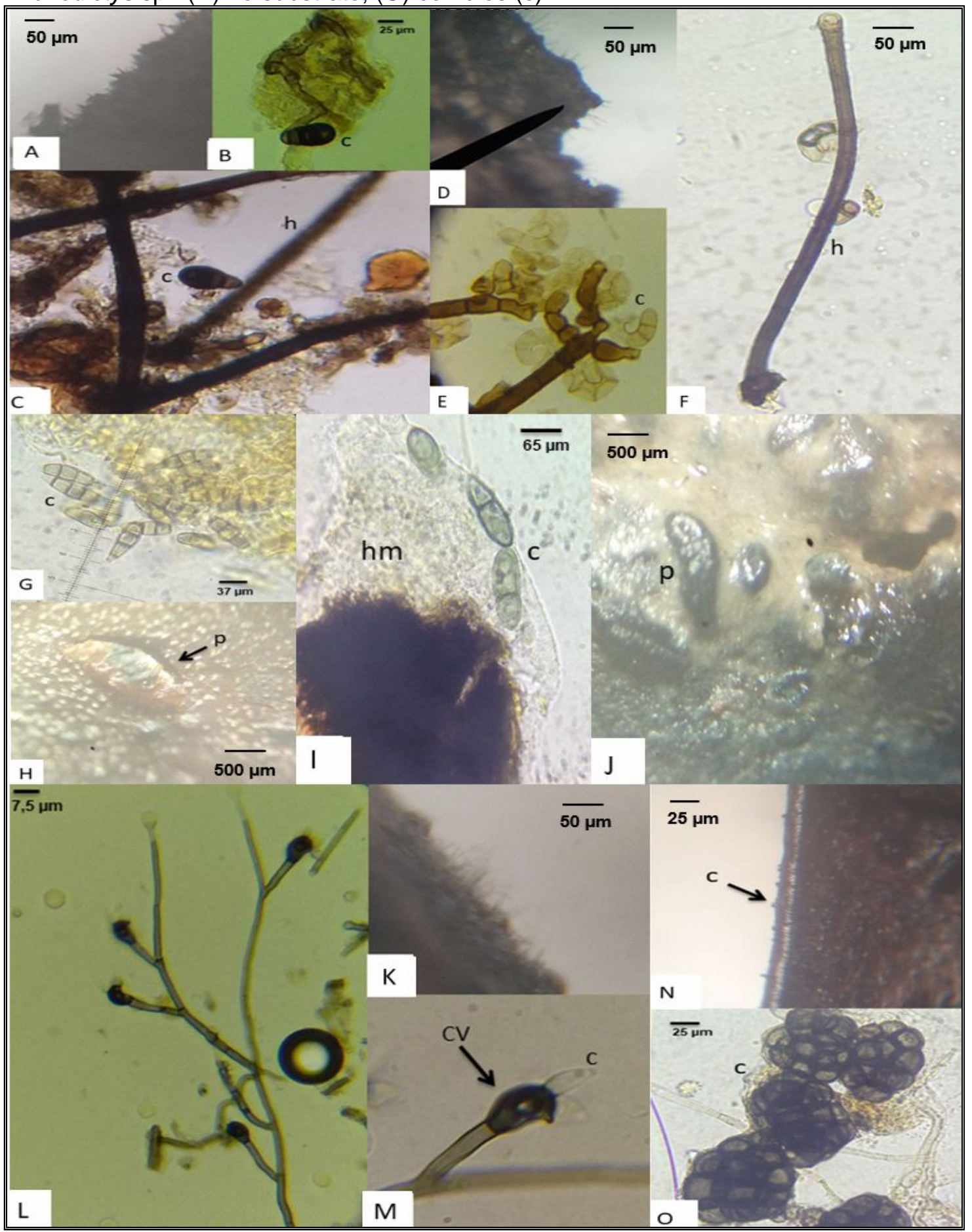




\section{Pestalotia}

Acérvulos escuros, discoides ou em forma de almofada, subepidérmicos, conidióforos curtos, simples, conídios dar, multicelulares, com células terminais hialinas, pontiagudas, elipsoide a fusoide, com dois ou mais apêndices hialinos, apicais, parasitas ou sapróbios (BARNETT; HUNTER, 1998). Pestalotia sp. encontrado tem o conídio de 7,5 $\mu \mathrm{m}$ de largura e comprimento de $42 \mu \mathrm{m}$, cinco septos sendo que os da extremidade são hialinos e os do meio são cinzas imerso em himênio (Figura 3).

\section{Zygosporium}

O gênero de hifomicetos Zygosporium é morfologicamente muito característico. Os conidióforos contêm uma vesícula geralmente única, escura e inchada que tem de duas a quatro paredes finas, células conidiogênicas claras. Cada célula conidiogênica dá origem a um solitário conídio. Os conídios variam de hialino a marrom, liso a ornamentado de várias maneiras. O gênero é predominantemente sapróbio, geralmente encontrado em folhas mortas de uma grande variedade de espécies de plantas, embora Z. majus seja parasita (ELLIS, 1971, 1976).

Zygosporium mycophilum encontrado na coleta tem conidióforo vesicular de largura de 7,5 $\mu \mathrm{m}$ e comprimento de $12,5 \mu \mathrm{m}$ preta, conídio com largura de 5,0 $\mu \mathrm{m}$ e comprimento de 7,5 $\mu \mathrm{m}$, hialino e hifas septadas acinzentadas a pretas (Figura 3).

\section{ANÁLISES ECOLÓGICAS}

As análises ecológicas mostraram que o gênero Bipolaris foi o que apresentou mais espécimes nas coletas. A maioria das espécies obtivera 33,3\% de constância no local amostrado, pois apareceram em apenas uma das coletas e Bipolaris sp. 1 obteve $66,7 \%$ de constância aparecendo em duas áreas diferentes (Tabela 2).

TABELA 2. Cálculo de Constância das espécies de microfungos identificadas.

\begin{tabular}{|c|c|c|c|c|}
\hline \multirow{2}{*}{ MICROFUNGOS } & \multicolumn{3}{|c|}{ COLETAS } & \multirow{2}{*}{$\mathbf{C} \%$} \\
\hline & 01 & 02 & 03 & \\
\hline Aspergillus niger & $\mathrm{X}$ & & & $33,3 \%$ \\
\hline Aspergillus sp. & $X$ & & & $33,3 \%$ \\
\hline Bipolaris crotonis & $X$ & & & $33,3 \%$ \\
\hline Bipolaris sp. 1 & $\mathrm{X}$ & & $\mathrm{X}$ & $66,7 \%$ \\
\hline Bipolaris sp. 2 & & & $\mathrm{X}$ & $33,3 \%$ \\
\hline Bactrodesmium sp. & & & & $33,3 \%$ \\
\hline Helicosporium sp. & & $\mathrm{X}$ & & $33,3 \%$ \\
\hline Monodictys sp. & & $\mathrm{X}$ & & $33,3 \%$ \\
\hline Nectria sp. & $\mathrm{X}$ & & & $33,3 \%$ \\
\hline Pestalotia sp. & $\mathrm{X}$ & & & $33,3 \%$ \\
\hline Zygosporium mycophilum & & & $\mathrm{X}$ & $33,3 \%$ \\
\hline Não Identificado 1 (NI 1) & $X$ & & & $33,3 \%$ \\
\hline Não Identificado 2 (NI 2) & $\mathrm{X}$ & & & $33,3 \%$ \\
\hline Não Identificado 3 (NI 3) & $\mathrm{X}$ & & & $33,3 \%$ \\
\hline Não Identificado 4 (NI 4) & & $X$ & & $33,3 \%$ \\
\hline Não Identificado 5 (NI 5) & & & $\mathrm{X}$ & $33,3 \%$ \\
\hline
\end{tabular}

De acordo com Dajoz (1978), as espécies que apareceram em $25 \%$ das coletas são classificadas como espécies acessórias e as que tiveram porcentagem maior que 
$50 \%$ são classificadas em espécies constantes. Portanto, a espécie Bipolaris sp. 1 está classificada como constante enquanto que as outras espécies identificadas são consideradas acessórias, visto que elas apareceram apenas uma vez em cada coleta.

De acordo com os cálculos de similaridade de Sorensen, não houve similaridade entre as áreas $\mathbf{A}$ e $\mathbf{C}$, apresentando valor máximo de diversidade de Whittakker. Comparando-se as áreas $\mathbf{A}$ e $\mathbf{B}$ e as áreas $\mathbf{B}$ e $\mathbf{C}$, também apresentaram baixa similaridade e alta diversidade (Tabela 3). A diversidade $\beta$ total de Whittakker obteve um valor de 2,7 que indica alto índice de diversidade entre todas as áreas estudadas.

TABELA 3. Resultados dos índices de Similaridade de Sorensen e Medidas $\beta$ de Whittakker entre as áreas de estudo

\begin{tabular}{ccc}
\hline ÁREAS & $\begin{array}{c}\text { SIMILARIDADE DE } \\
\text { SORENSEN }\end{array}$ & DIVERSIDADE DE WHITTAKKER \\
\hline Área A X Área B & $20 \%$ & 0,80 \\
Área B X Área C & $16,7 \%$ & 0,83 \\
Área A X Área C & $0,0 \%$ & 1,0 \\
\hline
\end{tabular}

A área A, que apresentou uma riqueza de seis espécies, é um local com características comuns de um manguezal preservado, com muitos indivíduos da fauna local, espécies arbóreas endêmicas do ecossistema, muita lama e parecendo ser menos antropizada. $\mathbf{A}$ área $\mathbf{B}$, que apresentou uma riqueza de quatro espécies, tem uma parte muito alagada, que mal se consegue entrar, uma parte com grande incidência de areia (bancos de areia) e muitas clareiras, sendo perceptível que algumas pessoas acampem por ali, pois um casal foi avistado em um dia das coletas, além de lixo ao seu redor, podendo, portanto, justificar o motivo dessa área ser a que menos obteve espécies. Também foi perceptível que a área $\mathbf{C}$ teve mais espécies encontradas $(R=8)$ e identificadas, isso pode ser explicado porque essa área fica mais próxima de um bairro e mesmo sofrendo com antropização há a introdução de outras espécies vegetais que não é endêmica de manguezal, como por exemplo, castanheiras (Tabela 4).

TABELA 4. Identificação dos microfungos e Riqueza $(R)$ de Espécies de microfungos das áreas estudadas no manguezal.

\begin{tabular}{|c|c|c|}
\hline $\begin{array}{c}\text { ÁREA A } \\
\text { (preservada) }\end{array}$ & $\begin{array}{c}\text { ÁREA B } \\
\text { (estressada) }\end{array}$ & $\begin{array}{c}\text { ÁREA C } \\
\text { (antropizada) }\end{array}$ \\
\hline $\begin{array}{c}\text { Aspergillus niger } \\
\text { Aspergillus sp. } \\
\text { Bipolaris crotonis } \\
\text { Bipolaris sp. } 1 \\
\text { Bactrodesmium sp. } \\
\text { Zygosporium mycophilium }\end{array}$ & $\begin{array}{l}\text { Bipolaris sp. } 1 \\
\text { Helicoma sp. } \\
\text { Monodictys sp. } \\
\text { Não Identificado } 1\end{array}$ & $\begin{array}{c}\text { Bipolaris sp. } 2 \\
\text { Monodictys sp. } \\
\text { Nectria sp. } \\
\text { Pestalotia sp. } \\
\text { Não Identificado } 2 \\
\text { Não Identificado } 3 \\
\text { Não Identificado } 4 \\
\text { Não Identificado } 5 \\
\end{array}$ \\
\hline$R=6$ & $R=4$ & $R=8$ \\
\hline
\end{tabular}

De acordo com Devadatha et al. (2021), diversos fatores determinam a 
biodiversidade fúngica de manguezal, tais como: tamanho da amostra; diversidade e tamanho do manguezal; disponibilidade de madeira em decomposição; natureza do tecido do hospedeiro; tempo de exposição à água do mar; amplitude de maré; sucessão no substrato; interação fúngicas; zoneamento vertical e horizontal de fungos; parâmetros físicos e químicos como pressão hidrostática, luz, efeitos osmóticos, nível de oxigênio, $\mathrm{pH}$, poluentes, salinidade e temperatura.

Neste estudo de funga, em substratos de um manguezal da região do Extremo Sul da Bahia, observou-se que este manguezal de Alcobaça-BA possui maior riqueza de espécies na área antropizada, que se encontra em um estágio de degradação em relação as outras duas áreas estudadas. De acordo com Penttilä et al. (2006), a fragmentação e perda de habitats podem influenciar negativamente a diversidade da funga, porém, isto não foi observado neste presente estudo, já que a área antropizada apresentou maior riqueza de espécies. Características climáticas e fitofisionômicas podem ter influenciado nestes resultados, pois estas variáveis eram diferentes nas três áreas.

Em outras pesquisas, como as de Jia-Long et al. (2016), também observaram baixa similaridade entre os fungos endofíticos em uma área de manguezal na China e pesquisas de Shearer et al. (2007) relataram a diversidade no manguezal analisando espécies de água doce e salgada e Costa et al. (2012), que fez análise do índice de diversidade de Shannon entre estação seca e chuvosa, observaram baixa similaridade entre ambas.

Como este estudo é pioneiro na região, mostra a importância de se conhecer cada vez mais sobre a funga existente neste importante Bioma que é o mangue. Além disso, áreas de manguezais vêm sendo constantemente ameaçadas de extinção devido a antropização e, consequentemente, importantes espécies de microfungos podem ser perdidos sem mesmo antes terem sido conhecidos.

\section{CONCLUSÃO}

Foram registradas 10 espécies de microfungos em nível de gêneros na área de estudo, três em nível de espécies e cinco não identificados, sendo encontrados em cinco diferentes tipos de substratos presentes no ecossistema de Manguezal, como galhos, folhas, flor, cascas e frutos.

As análises ecológicas apontaram que há diversidade significativa e a quantidade de espécies em cada área pode ser explicada pela caracterização de cada uma, isto é, há influência da biodiversidade fúngica em relação ao nível de preservação do meio ambiente do tipo manguezal.

É nítido, nos vários âmbitos da ciência, que as pesquisas sobre fungos vêm crescendo e que não devem parar, pois, ainda há muito a se descobrir, seja na área das ciências ambientais, médicas, alimentícia, etc., principalmente na região do Extremo Sul da Bahia na qual os estudos ainda são recentes. Portanto, espera-se, com esse estudo, conhecer um pouco mais dos fungos da região e que este possa proporcionar o incentivo para mais pesquisas sobre a funga de manguezal. 


\section{REFERÊNCIAS}

ALMEIDA, É. A.; SANTANA, J. S.; FORTUNA, J. L. Microfungos em fragmento de Mata Atlântica no Extremo Sul da Bahia. Enciclopédia Biosfera, v. 17, n. 34, p. 328-343, 2020. URL: https://conhecer.org.br/ojs/index.php/biosfera/article/view/2083

ALONGI, D. M. Present state and future of the world's mangrove forests. Environmental Conservation, v. 29, p. 331-349, 2002 . DOI: https://doi.org/10.1017/S0376892902000231

BARNETT, H. L.; HUNTER B. B. Illlustrated Genera of Imperfect Fungi. St. Paul, Minnessota, 1998, $237 \mathrm{p}$.

BEGON, M.; TOWNSEND, C. R.; HARPER, J. L. Ecologia. De Indivíduos a Ecossistemas. 4 ed. Porto Alegre: Artmed. 2007. 752 p.

BRASIL. Ministério do Meio Ambiente, Instituto Chico Mendes de Conservação da Biodiversidade. Atlas dos Mangues do Brasil. Brasília, 2018. 179 p.

BROWER, J. E.; ZAR, J. H.; VON ENDE, C. A. Field and Laboratory Methods for General Ecology. 4 ed. Dubuque: C. Brown Publishers. 1998.

CAI, L.; HYDE, K. D.; TSUI, C. K. M. Genera of Fleshwater Fungi. Hong Kong: Fungal Diversity Press. June. 2006. 261 p.

CAMPOS, E. L.; SOTÃO, H. M. P.; CAVALCANTI, M. A. Q.; LUZ, A. B. Basidiomycetes de manguezais da APA de Algodoal-Maiandeua, Pará, Brasil. Boletim do Museu Paraense Emílio Goeldi. Série Ciências Naturais, v. 1, n. 1, p. 141-146, 2005. URI: http://repositorio.museu-goeldi.br/handle/mgoeldi/530

CARVALHO, L. I. C. Aspergillus e Aspergilose - Desafios no combate da doença. Dissertação (Mestrado). Universidade Fernando Pessoa. Mestrado Integrado em Ciências Farmacêuticas. Porto, 2013.

CASTAÑEDA, R. F.; HEREDIA, G.; GUSMÃO, L. F. P.; LI, D. Fungal Diversity of Central and South America. Chapter 9. p. 197-217. In: LI, D. Biology of Microfungi. New York: Springer. 2016, 651 p.

CASTELLANI, A. Viability of some pathogenic fungi in distilled water. Journal of Tropical Medicine and Hygiene. v. 42, p. 225-226, 1939.

CHENG, Z. S.; PAN, J. H.; TANG, W. C.; CHEN, Q. J. LIN, Y. C. Biodiversity and biotechnological potential of mangroveassociated fungi. Journal of Forestry Research, v. 20, p. 63-72, 2009. URL: https://link.springer.com/article/10.1007/s11676-009-0012-4

COSTA, I. P. M. W.; MAIA, L. C.; CAVALCANTI, M. A. Diversity of leaf endophytic fungi ENCICLOPÉDIA BIOSFERA, Centro Científico Conhecer - Jandaia-GO, v. 18 n.35; p. 109 
in mangrove plants of northeast Brazil. Brazilian Journal of Microbiology, v. 43, n. 3, p. 1.165-1.173. 2012. DOI: https://doi.org/10.1590/S1517-83822012000300044

DAJOZ, R. Ecologia Geral. 3 ed. Petrópolis: Vozes, p. 474. 1978.

DEBBAB, A; ALY, A. H.; PROKSCH P. Mangrove derived fungal endophytes-a chemical and biological perception. Fungal Diversity, v. 61, p. 1-27, 2013. URL: https://link.springer.com/article/10.1007/s13225-013-0243-8

DEVADATHA, B.; JONES, E. B. G.; PANG, K. L.; ABDEL-WAHAB, M. A.; HYDE, K. D.; et al.,; Occurrence and geographical distribution of mangrove fungi. Fungal Diversity, s.n., n.p., 2021. DOI: https://doi.org/10.1007/s13225-020-00468-0

DUAN, J.; WU, W.; LIU X. Z. Dinemasporium (Coelomycetes). Fungal Diversity. v. 26, p. 205-208, 2007. URL: https://www.fungaldiversity.org/fdp/sfdp/26-8.pdf

ELLIS, M. B. Dematiaceous Hyphomycetes. Surrey: Commonwealth Mycological Institute. 1971. 608 p.

ELLIS, M. B. More dematiaceous hyphomycetes. Kew, Surrey, commonwealth Mycological institute. 1976. 507 p.

EWEL, K. C.; TWILLEY, R. R.; ONG, J. E. Different kinds of mangrove forests provide different goods and services. Global Ecology and Biogeography, v. 7, p. 83-94, 1998. DOI: https://doi.org/10.1111/j.1466-8238.1998.00275.x

GUSMÃO, L. F. P. Fungos. Cap. 3. p. 47-57. In: FUNCH, L. S.; MIRANDA, A. P. Serrano, Parque Municipal da Muritiba. Feira de Santana: Print Mídia. 2011. 188 p.

GUSMÃO, L. F. P.; GRANDI, R. A. P. Hyphomycetes com conidioma dos tipos esporodóquio e sinema associados a folhas de Cedrela fissilis (Meliaceae), em Maringá, PR, Brasil. Acta Botânica Brasilica. v. 11, n. 2, p. 126-134, 1997. DOI: https://doi.org/10.1590/S0102-33061997000200004

GUSMÃO, L. F. P.; PEFNNING, L. Incertaesedis 2015. Flora do Brasil. 2020 em construção. Jardim Botânico do Rio de Janeiro. [online]. Disponível em: <http://floradobrasil.jbrj.gov.br/jabot/floradobrasil/FB93068> Capturado em 01 de janeiro de 2018.

JIA-LONG, L.; XIANG, S.; LIANG, C.; LIANG-DONG, G. Community structure of endophytic fungi of four mangrove species in Southern China. Mycology, v. 7, n. 4, p. 180-190, 2016. DOI: https://doi.org/10.1080/21501203.2016.1258439

JONES, E. B. G.; ALIAS, S. A. Biodiversity of Mangrove Fungi. Biodiversity of Tropical Microfungi, v. 6, p. 71-92, 1997. 
KATHIRESAN, K. A review of studies on Pichavaram mangrove, southeast India. Hydrobiologia, v. 430, p. $185-205, \quad 2000 . \quad$ URL: https://link.springer.com/article/10.1023/A:1004085417093

LACERDA, E. R.; JAKOVAC, A. C. C. Mangues. Aliados contra as mudanças climáticas. Ciência Hoje, n. 370, p. 48-54, 2020. URL: https://cutt.ly/Gj7R5MP

MAGURRAN, A. E. Medindo a Diversidade Biológica. Curitiba: Ed. UFPR. 2013, 261 p.

MANAMGODA, D. S.; ROSSMAN, A. Y.; CASTLEBURY, L. A.; CROUS, P. W.; CHUKEATIROTE, H. E.; HYDE, K. D. The genus Bipolaris. Studies in Mycology, v. 79, p. 221-288, 2014. DOI: https://doi.org/10.1016/j.simyco.2014.10.002

MARTINS, J. E. C.; MELO, N. T.; HEINS-VACCARI, E. M. Atlas de Microbiologia Média. São Paulo: Manole. 2005.

MULLER-DOMBOIS, D. Ecological measurements and microbial populations. p. 173184. In: WICKLOW, D. T.; CARROL, G. C. (Eds.). The Fungal Community: Its Organization and Role in the Ecosystem. New York: Marcel Derker. 1981.

NAGELKERKEN, I.; VAN DER VELDE, G.; GORISSEN, M. W.; MEIJER, G.J. VAN'T HOF, T.; DEN HARTOG, C. Importance of mangroves, seagrass beds and the shallow coral reef as a nursery for important coral reef fishes, using a visual census technique. Estuarine, Coastal and Shelf Science, v. 51, p. 31-44, 2000. DOI: https://doi.org/10.1006/ecss.2000.0617

ODUM, E. P. Ecologia. Rio de Janeiro: Guanabara Koogan. 1988. 434 p.

ODUM, E. P. Fundamentos de Ecologia. 6 ed. Lisboa: Fundação Calouste Gulbenkian. 2001. 927 p.

OLIVEIRA, H. V. V.; FORTUNA, J. L. Microfungos em fitotelmata de bromélias de um fragmento de Mata Atlântica do Extremo Sul da Bahia. Unisanta BioScience, v. 9, n. 4, p. 338-355, 2020. URL: https://periodicos.unisanta.br/index.php/bio/article/view/2599/1896

PENTTILÄ, R.; LINDGREN, M.; MIETTINEN, O.; RITA, H.; HANSKI, I. Consequences of forest fragmentation for polyporous fungi at two spatial scales. Oikos, v. 114, p. 225240, 2006. DOI: https://doi.org/10.1111/j.2006.0030-1299.14349.x

PINTO-COELHO, R. M. Fundamentos em Ecologia. Porto Alegre: Artmed. 2000. 252 p. 
RICKLEFS, R. E. A Economia da Natureza. 5 ed. Rio de Janeiro: Guanabara Koogan. 2003. 503 p.

SCHAEFFER-NOVELLI, Y. Manguezal: Ecossistema Entre a Terra e o Mar. São Paulo. Caribbean Ecological Research, 1995. 64 p.

SEIFERT, K.; MORGAN-JONES, G.; GAMS, W.; KENDRICK, B. The Genera of Hyphomycetes. Utrecht: CBS-KNAW. 2011. 997 p.

SHEARER, C. A.; DESCALS, E.; KOHLMEYER, B.; KOHLMEYER, J.; MARVANOVÁ, L.; et al.,; Fungal biodiversity in aquatic habitats. Biodiversity and Conservation, v. 16, p. 49-67, 2007. URL: https://link.springer.com/article/10.1007/s10531-006-9120-z

SILVA, J. C. Importância econômica e biotecnológica do guaiamum (Cardisoma guanhumi, Latreille, 1828) (Brachyura: Gecarcinidae) em Alcobaça, Bahia. Teixeira de Freitas: Universidade do Estado da Bahia (UNEB), 2018. 59 f. Trabalho de Conclusão de Curso (Monografia). Área de concentração: Carcinologia.

SILVA, T. N.; FORTUNA, J. L. Microfungos associados a substratos em fitotelmata de bromélias de um fragmento de Mata Atlântica no Extremo Sul da Bahia. Anais... I Semana de Biologia da Universidade Federal do Sul da Bahia, Porto Seguro-BA, p 1617. 2019.

VIANA, C. G.; FORTUNA, J. L. Microfungos endofíticos e epifíticos em folhas de bromélias em um fragmento de Mata Atlântica no Extremo Sul da Bahia. Unisanta BioScience, v. 9, n. 4, p. 356-370, $2020 . \quad$ URL: https://periodicos.unisanta.br/index.php/bio/article/view/2600/1895

WIJAYAWARDENE, N. N.; HYDE, K. D.; WANASINGLE, D. N.; PAPIZADEH, M.; GOONASEKARA, I.; et al.,;Taxonomy and phylogeny of dematiaceous coelomycetes. $\begin{array}{llllll}\text { Fungal Diversity. } & \text { v. } & 77, & 2016 . & 316 & \text { p. }\end{array}$ https://link.springer.com/article/10.1007/s13225-016-0360-2 